\title{
Construction and Test of Full-Size Micromegas Modules for the ATLAS New Small Wheel Upgrade
}

\author{
Jonathan Bortfeldt ${ }^{1}$, a on behalf of the ATLAS Muon Collaboration \\ ${ }^{1}$ Ludwig-Maximilians-University Munich, Germany
}

\begin{abstract}
In 2015 the first full size resistive-strip Micromegas modules for the ATLAS New Small Wheel upgrade will be realized. The goal is to provide precision muon tracking with spatial resolution below $100 \mu \mathrm{m}$ on trapezoidal four-layer detector modules with areas between 2 and $3 \mathrm{~m}^{2}$. This poses stringent limits on the overall accuracy of the modules with respect to strip positioning and planarity. Each module is built as a quadruplet of four resistive strip Micromegas layers, constructed from stiff sandwich panels. An assembly procedure has been developed to build the panels and modules with the required mechanical precision of $30 \mu \mathrm{m}$ along the precision coordinate and $80 \mu \mathrm{m}$ perpendicular to the chamber. The construction and quality assurance procedures ensure good module quality verified by checks done during the construction and the chamber assembly.
\end{abstract}

\section{Introduction}

ATLAS is a multi-purpose detector at the Large Hadron Collider (LHC) at CERN [1] with almost full solid angle coverage. The muon system represents the outermost layer of the detector. It consists of separate tracking and triggering chambers in toroidal magnetic fields, enabling a standalone muon momentum measurement [2]. Monitored Drift Tube Chambers (MDT) are used for tracking in the barrel and the endcap region. Only in the central region of the endcap station, in the so called Small Wheel, that is closest to the interaction point, Cathode Strip Chambers are used. Resistive Plate Chambers provide the trigger in the barrel and Thin Gap Chambers in the endcap region of the muon spectrometer.

The instantaneous luminosity of the LHC will be increased from its design value of $\mathcal{L}=1 \times 10^{34} \mathrm{~cm}^{-2} \mathrm{~s}^{-1}$ up to $\mathcal{L}=7 \times 10^{34} \mathrm{~cm}^{-2} \mathrm{~s}^{-1}$ in 2026 . The background hit rates by photons and neutrons increase accordingly and can reach up to $15 \mathrm{kHz} / \mathrm{cm}^{2}$ in the forward region of the muon spectrometer. In order to preserve the current muon triggering capabilities and momentum determination accuracy, the muon detectors in the Small Wheel need to be replaced.

Small strip Thin Gap Chambers (sTGC) have been chosen as main trigger and resistive strip Micromegas as main tracking detector [3], where each detector technology is capable of providing both functionalities. Each New Small Wheel consists of 8 large and 8 small sectors. Each sector consists of $2 \times 4$ layers of sTGCs, sandwiching $2 \times 4$ layers of Micromegas, mounted on a spacer frame. Each Micromegas part of a sector consists of upper and lower quadruplet chambers. The dimensions of

\footnotetext{
ae-mail: jonathan.bortfeldt @ cern.ch
}

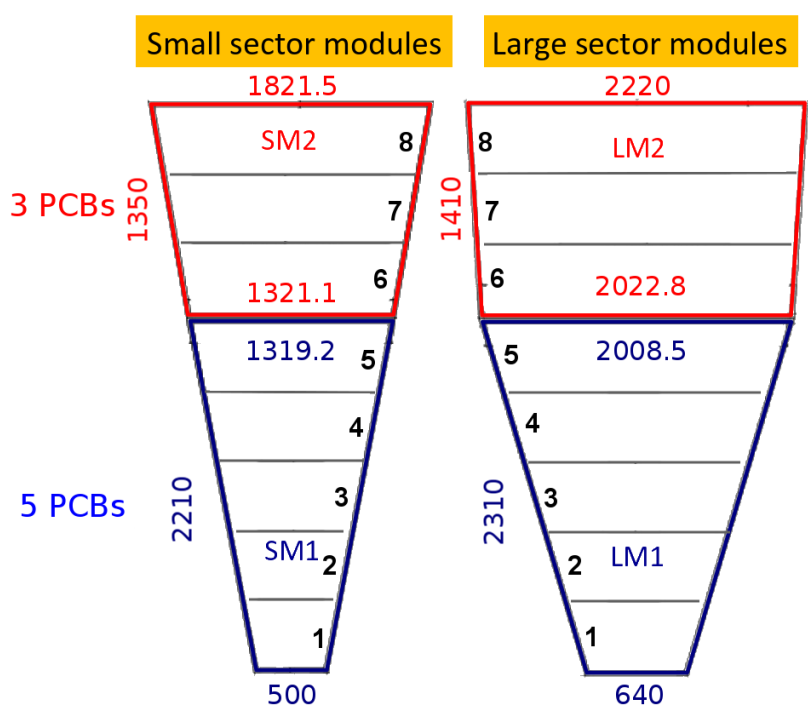

Figure 1. Dimensions of the small and large New Small Wheel Micromegas sectors in millimeters. Each Micromegas chamber consists of three or five printed circuit boards (PCB) per layer, numbered from 1 to 5 and 6 to 8 .

these chambers are shown in figure 1. Four modules differing in dimension are constructed by the participating institutes in Italy (SM1), Germany (SM2), France (LM1) and Russia/Greece/CERN (LM2). Two layers of each Micromegas quadruplet are rotated with respect to the other two by $\pm 1.5^{\circ}$ such that these stereo layers provide a second coordinate hit measurement with millimeter accuracy.

In this paper, the construction and quality control procedures for the first full size Micromegas modules of the New Small Wheel are described and discussed. 


\section{Micromegas Chambers for the ATLAS New Small Wheel}

\subsection{Resistive Strip Micromegas}

A resistive strip Micromegas is shown schematically in figure 2. The copper readout strips are covered by a congruent layer of screen-printed resistive strips. These anode strips are insulated with respect to the readout strips by a $50 \mu \mathrm{m}$ thick Kapton foil and are supplied with highvoltage. This scheme suppresses the influence of discharges between anode and mesh on the detection efficiency [4], that are potentially created by strongly ionizing background particles. The micro-mesh is carried by a pre-

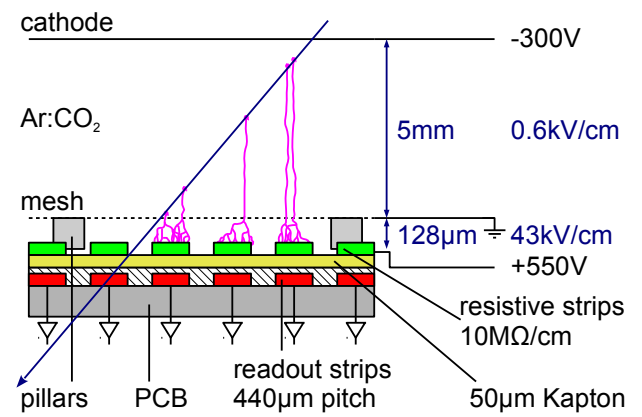

Figure 2. Schematic view of a resistive strip Micromegas.

cise aluminum frame, that is permanently attached to the cathode structure. This facilitates cleaning of the amplification region between mesh and anode during assembly and thus simplifies the commissioning. The readout strips are individually connected to the readout electronics using solderless connectors.

Each readout printed circuit board (PCB) carries several copper precision markers and chessboardlike coded alignment markers, so called RasNiK masks [5], a development used extensively for monitoring ATLAS muon detectors. They are produced in the same step as the readout strips and thus have a precise relative alignment with respect to the readout structure. Measuring the position of the circular or long-hole precision markers or the RasNiK masks with telecentric optics or contact-CCDs allows for a precise optical positioning of the readout structures and the final panels during assembly.

\subsection{Module Design and Accuracy}

A schematic view of a Micromegas quadruplet is shown in figure 3. The overall thickness of each module is about $70 \mathrm{~mm}$ and the total number of readout channels is on the order of $1.5 \cdot 10^{4}$ per module. It is constructed from two readout panels with readout anodes on both sides and three drift panels, that carry the cathode structures. The readout structure consists of strips with $0.43 \mathrm{~mm}$ pitch and up to $2 \mathrm{~m}$ length. Readout and cathode PCBs are used as faces of glued sandwich panels, consisting of aluminum honeycomb and aluminum reinforcement bars. The two outer drift panels are identical and carry cathode structure and micro-mesh on the inner side. The central double sided

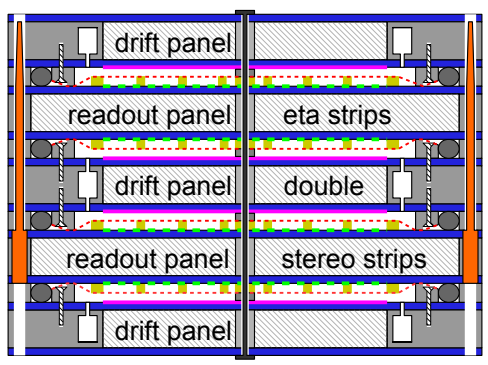

Figure 3. Schematic view of Micromegas quadruplet (not to scale). The cathode structure (magenta) is carried by three drift panels, the readout structure (green) is supported by two double readout panels. The micro-mesh (red) is attached to an aluminum frame, supported by the drift panels, its distance to the readout structure is defined by $128 \mu \mathrm{m}$ high insulating pillars (yellow).

drift panel carries cathode and mesh on both sides. The two double sided readout panels carry the parallel etaand the rotated stereo strips, respectively. The five panels are screwed together during assembly and sealed with O-rings, such that an opening of the chamber for maintenance is possible, if needed. Interconnections between the five panels minimize the deformation of the chamber, induced by a slight overpressure due to the continuous flushing of the chamber with detector gas.

The desired momentum resolution of $10 \%$ for muons with $1 \mathrm{TeV}$ transverse momentum can be reached by chambers capable of measuring the overall muon track position with an accuracy better than $50 \mu \mathrm{m}$. This requirement can be translated into accuracy requirements (RMS) for the readout and cathode structure of a quadruplet for controlled temperature conditions. The absolute positioning of readout strips must be better than $35 \mu \mathrm{m}$, the relative alignment of the readout PCBs on one side of a panel and the relative alignment of the two sides of a readout panel must be better than $25 \mu \mathrm{m}$. Finally the relative alignment of the two readout panels should be better than $18 \mu \mathrm{m}$. The surface of readout and drift panel should be planar within $37 \mu \mathrm{m}$. During operation in the New Small Wheel the alignment of the chambers will be monitored with an optical system.

\subsection{Concept of Panel Construction}

The idea of panel construction is illustrated in figure 4 . The PCBs are sucked upside-down against a granite table with a maximum deviation from planarity smaller than $10 \mu \mathrm{m}$. Thus the table planarity transfers to the PCB. $10 \mathrm{~mm}$ thick aluminum honeycomb and aluminum bars are

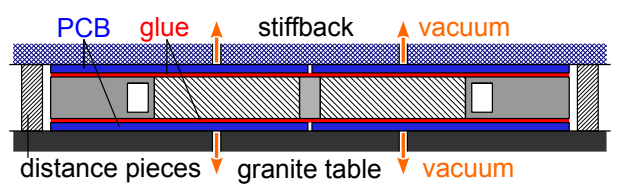

Figure 4. Panel construction on a planar granite table. 
then glued by a $200 \mu \mathrm{m}$ thick layer of Araldite 2011. The PCBs of the second face, with an equally thick layer of glue, are placed on top. During glue curing, the second side is sucked against a precise but light-weight sandwichtable, called stiffback, thus ensuring planarity of the second side. The parallelism of both sides is ensured by precision distance pieces between granite table and stiffback.

Two different gluing techniques are used at the contributing sites: The discussed stiffback method, when accurate absolute positioning of the two panel faces is required and alternatively a vacuum bag method, described in section 3. Also, two different drilling schemes are used: Drilling and milling of all holes and threads into the individual components before panel assembly or alternatively machining after gluing in a single step. Since the assembly techniques for SM1 and LM2 and for SM2 and LM1 are similar, the panel construction is discussed exemplarily for the SM1 and SM2 modules in sections 3 and 4.

\section{Drift Panel Construction}

The drift panels of the SM1 modules are assembled using the vacuum bag method, gluing both sides simultaneously. All machining is done prior to panel assembly. Each of the five drift PCBs features a pair of precision hole and slot. An aluminum bar with several removable precision dowel pins is mounted on the granite table. Glue is distributed on the PCBs before transferring them to the granite table. The upper PCB (number 5 in figure 1) is aligned with respect to the aluminum bar using two precision pins in the hole and slot of the PCB. The remaining four PCBs are then aligned relative to the upper using feeler gauges to define the distance between boards and a single precision pin in each slot. The aluminum honeycomb and the aluminum bars are placed into the glue and aligned relative to the PCBs using dowel pins in the mounting holes. This arrangement is shown in figure 5. The second layer of PCBs

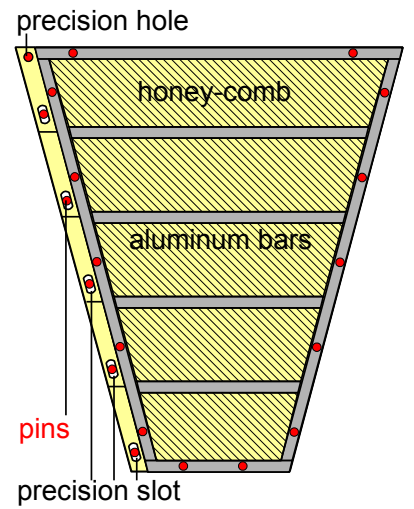

Figure 5. Open SM1 drift panel, before closing with the second layer of PCBs, which are pressed against the sandwich with a vacuum bag during glue curing.

is then placed on top, a vacuum bag covers the sandwich and the air is pumped out of the sandwich, pressing the components against the granite table. This method profits from the manufacturing precision of the thickness of PCBs, honeycomb and bars.

The SM2 drift panels are glued with the same method as the SM2 readout panels, c.f. section 4.2.

\section{Readout Panel Construction}

\subsection{SM1 Module}

While the lower side lies on a granite table, the upper side of the SM1 readout panels is supported by a stiffback during curing, both sides are glued simultaneously and all machining is done prior to gluing. The granite table and the stiffback permanently carry precision aluminum plates with milled vacuum suction channels and precision holes. Removable dowel pins fit into the precision holes. The readout PCBs and bars are aligned with respect to the aluminum plates using the dowel pins. The relative position of the two panel sides during glue curing is ensured by a tapered and a $\mathrm{V}$-shaped interlock attached to the aluminum plates on the table and on the stiffback.

\subsection{SM2 Module}

The internal alignment of the SM2 module is performed relative to two steel cylinders with precise distance. Onto the reverse side of each readout PCB a brass slot and a brass hole washer are glued, concentric to the two precision markers, c.f. section 2.1. Alignment with an accuracy below $5 \mu \mathrm{m}$ is achieved during adjustment when observing the washer and the precision marker with a telecentric camera. The three readout PCBs are then placed onto a granite table. A stiff aluminum alignment frame, carrying six precision dowel pins is placed on top of the three boards. The dowel pins fit into the hole and slot washers on the readout boards and thus ensure the precise relative alignment of the three boards. The alignment frame additionally carries two precision surfaces, straight and V-shaped. Pressing these against two cylinders, permanently mounted on the granite table, ensures the accurate absolute positioning of the readout PCBs, see figure 6 . After sucking the three boards against the granite table,

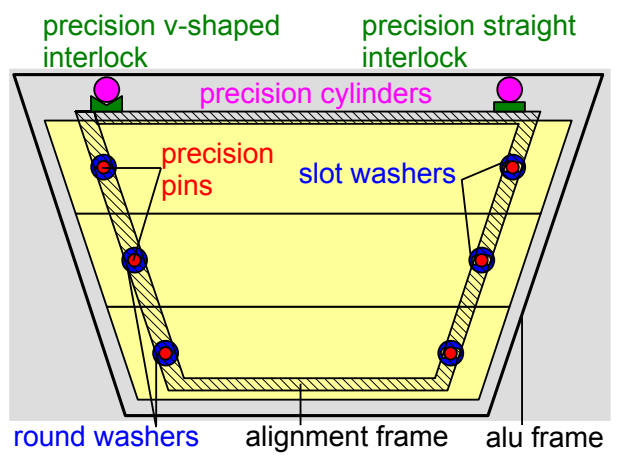

Figure 6. Readout PCBs (yellow) on the granite table, precisely aligned with respect to two cylinders (magenta) by a removable alignment frame (hatched).

the alignment frame can be removed. A V-shaped and a straight precision piece is glued onto the upper PCB, each precisely aligned with respect to the two cylinders. Aluminum honeycomb and bars are glued on the boards relative to a fixed aluminum frame using distance pieces. After glue curing, this semi-panel is removed and transferred to 
a stiffback. The alignment procedure on the granite table is repeated for the second PCB layer and the semi-panel, sucked against the stiffback, is glued onto it.

The readout panel is finally transferred to a large CNC machine, where all holes and threads are machined. All precision holes are manufactured in a single step without moving the panel, leading to an excellent relative alignment with machine precision.

\subsection{Alignment Structure Gluing}

The critical alignment between the two readout panels is achieved mechanically (figure 7). Two precision pins are glued into the stereo strip readout boards (c.f. figure 3) after glue curing for the SM1 panels and after milling for the SM2 panels, without moving the respective panel. Into

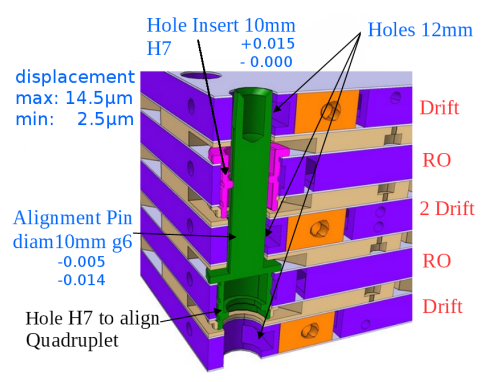

Figure 7. Two $10 \mathrm{~mm}$ alignment pins (green) with $\mathrm{g} 6$ precision in the stereo strip readout boards fit into matching slot and hole inserts (magenta) with $\mathrm{H} 7$ precision in the eta strip readout boards.

the eta strip readout boards two matching hole and slot inserts are glued consistently.

\section{Quality Assurance}

Many quality checks are performed on raw material and PCBs before panel assembly. Before and after panel gluing, the absolute positioning of the readout PCBs is confirmed optically. During module assembly, repeated highvoltage stability tests ensure the cleanliness of the detectors. In the following panel planarity and gas tightness measurements are discussed.

\subsection{Planarity Measurements}

Coordinate measurement machines equipped with tactile or with laser distance sensors are used to investigate the achieved planarity and parallelism of both readout panel sides after panel assembly. The accuracy of these systems is better than $10 \mu \mathrm{m}$. For three dummy panels the following planarities and min-max-deviations have been reached: $39 \mu \mathrm{m}$ and $200 \mu \mathrm{m}$ for a SM1 drift panel, $33 \mu \mathrm{m}$ and $200 \mu \mathrm{m}$ for a SM1 readout panel and $29 \mu \mathrm{m}$ and $190 \mu \mathrm{m}$ for a SM2 readout panel. The panels fulfill the ATLAS requirements.

\subsection{Gas Tightness Measurements}

The gas tightness of all panels is tested prior to module assembly with local and global methods. They rely on a measurement of the pressure drop as a function of time.
Proper sealing of joints between adjacent PCBs and at interconnects is confirmed. The overall tightness of panels is tested for each side individually in a full-size vessel.

\section{Mesh Stretching, Cleaning and Gluing}

Woven stainless-steel meshes with 250 lines/inch and $30 \mu \mathrm{m}$ wire diameter are stretched on pneumatic or mechanic clamps with a tension of $10 \mathrm{~N} / \mathrm{cm}$ and glued to transfer frames. The meshes are cleaned with solvent and high-pressure deionized water and finally glued onto precision aluminum bars, screwed onto the drift panels. The residual mesh is cut off.

\section{Quadruplet Assembly}

Modules are assembled vertically in a clean room to avoid dust contamination. The relative alignment of the two readout panels is ensured by two pairs of precision pins and bushings, c.f. figure 7. For the SM2 modules an alternative alignment, based on the $\mathrm{V}$-shaped and straight precision surfaces is foreseen. For the drift panels, an alignment with mounting hole accuracy is sufficient. After closing a final high-voltage test is performed, where each amplification structure is ramped up to almost $1 \mathrm{kV}$ under normal air atmosphere. In case of frequent discharges, the respective layer is reopened and cleaned.

The completed quadruplets are tested with cosmic rays to validate the overall accuracy and confirm the desired detection efficiency and operational stability. Using drift tube chambers as cosmic muon track reference, the position and potential deformation of the readout strips can be measured with an accuracy of $20 \mu \mathrm{m}$ [6].

\section{Conclusions}

The construction of quadruplet resistive strip Micromegas chambers with $30 \mu \mathrm{m}$ spatial resolution in the precision direction for the forward ATLAS muon spectrometer is challenging. The assembly and quality assurance procedures are reviewed and described. The desired planarity is reached. During the next few months, the first four fullsize quadruplet modules will be built with the desired precision.

\section{References}

[1] ATLAS Collaboration, JINST 3, S08003 (2008)

[2] ATLAS Collaboration, CERN-LHCC-97-022, CERN (1997)

[3] ATLAS Collaboration, CERN-LHCC-2013-006, CERN (2013)

[4] T. Alexopoulos et al., Nucl. Instr. and Meth. A 640, 110 (2011)

[5] H. van der Graaf et al., RasNiK, an Alignment System for the ATLAS MDT Barrel Muon Chambers, NIKHEF ETR94-10 (1994)

[6] Ph. Lösel et al., Nucl. Instr. and Meth. A (2015) 